d. Along similar lines the Women in Hypertension Research Committee has ensured that there are an abundance of opportunities to network and showcase work in this area. There will be several sessions throughout the programme highlighting cardiovascular disease in women, as well as awards and networking opportunities.

2.I am very pleased to announce that the ISH is now involved with two free online training platforms for GPs, nurses, pharmacists, community health workers and implementers. The Society first joined forces with the Omron Academy https://omron.platform.co.nl/\#/. In February 2020 a new platform with interactive learning experiences will also be launched where the ISH collaborated with other organisations and the Johns Hopkins Bloomberg School of
Public Health. The platform, titled Fundamentals for Implementing a Hypertension Program in Resource-Constrained Settings is freely available from the ISH website, and also here: https://globalhypertensionathopkins.org/ I firmly believe that such programmes are essential in improving informed decision making and task sharing particularly in low- and middleincome countries where significant intervention is required.

I trust you will once again enjoy our Newsletter. I want to personnally thank Lars Lindholm and the Editorial Team for their energy and continued efforts in making our Newsletter a delightful read!

See you in Glasgow!

With my very best wishes,

\title{
THE SECRETARY'S VOICE
}

\author{
THOMAS UNGER \\ CARIM - School for Cardiovascular Research, \\ Maastricht University, Maastricht, The Netherlands
}

DOI: $10.30824 / 2003-4$

The British philosopher and mathematician Alfred North Whitehead (1861-1947) has left us the following statement on the problem of translation: "I have often noticed that, if in an assembly of great scholars the topic of translations be introduced, they function as to their emotions and sentiments in exactly the same way as to decent people in the presence of a nasty sex problem. A mathematician has no scholastic respectability to lose..." (in: The Aims of Education, 1929), and in another essay in the same book: "Imagination is a contagious disease. It cannot be measured by the yard or weighed by the pound, and then delivered to the students by the faculty. It can only be communicated by a faculty whose members themselves wear their learning with imagination."

Seems that AN Whitehead didn't like the translationists very much and, of course, his statements did not refer to translational medicine, but I think they bear some general truth. Here we are: on one side those of us who see the only benefit of biomedical research in translating research results into clinical practice (translational medicine: from bench to bedside), on the other side those who strive imagination for curiosity per se. Further down 
in this issue of "Hypertension News" I try to discuss the apparent dichotomy which can be overcome through integration of both concepts into a common strategy.

When it comes to hypertension research and the development of new antihypertensive drugs, it is quite reasonable to ask for more imagination. The complex pathological issue that we call hypertension has been a research topic for decades, yet, if we want to explain the etio-pathology of the 'hypertension disease' to our students, we are lost in details of vascular, endocrine, nervous, renal, cardiac dysfunction without being able to put all the pieces of the puzzle together.

We desperately need more curiosity, more imaginationinhypertensionresearchtoopen up new horizons.

A European initiative with scholars from the universities of Maastricht, Glasgow, Madrid, Padua and Paris, aiming at the formation of a "European Hypertension School", is trying to 'shift the minds', i.e. to transcend existing standards of doctoral training in hypertension research by unifying vascular and endocrine approaches and establishing the dynamics of this network. A hopeful beginning.
New, imaginative approaches are also badly needed in the development of new antihypertensive drugs. The enormous translational success over the last seven decades beginning with sympatholytics up to the inhibitors of the RAAS has blinded us giving rise to the contention that translation from research to clinical use has done its job perfectly well and that novel antihypertensives are not necessary anymore. Indeed, the problem of lowering elevated blood pressure has been solved in most cases, undoubtedly an enormous success; however, the issue of the 'hypertensive disease' with all its complex pathological ramifications still needs to be addressed. The interplay of the pathological undercurrents such as genetics, inflammation, fibrosis, immunity in the context of neuronal, endocrine renal, vascular and cardiac dysfunction requires the development of treatment strategies beyond the mere blood pressure reduction. This has already begun with the RAAS inhibitors with their beneficial effects on some of the hypertensionrelated pathologies but needs to be followed by new, more comprehensive drug targets. The recent development of the SGLT2-Inhibitors, dealing with diabetes and hypertension at the same time, is a good example telling us where to go.

In the end, imagination or curiosity and translation are not necessarily mutually exclusive entities in hypertension research and the development of antihypertensives: They need each other to progress our understanding of the hypertensive disease in all its complexity and to help us developing drugs against novel cardiovascular/metabolic targets.

Thomas Unger - thomas.unger@maastrichtuniversity.nl

ISH 2020 GLOBAL HYPERTENSION PRACTICE GUIDELINES

\section{The International Society of Hypertension (ISH) is delighted to announce the release of the 2020 ISH Global Hypertension Practice Guidelines}

These new Guidelines are unique: In line with the mission of ISH to provide global assistance in our fight against hypertension, they divide for the first time diagnostic and treatment procedures into OPTIMAL (for the affl ent regions) and ESSENTIAL (for low resource settings) standards of care. Optimal care refers to evidencebased standards of care articulated in recent guidelines, whereas essential standards recognize that optimal

standards would not always be possible. Hence essential standards refer to minimum standards of care.

We are convinced that these new hypertension guidelines will benefit health providers and patients and help to raise the standards of hypertension management around the world.

The new ISH Hypertension Guidelines will be presented at the upcoming ESH/ISH Scientific Meeting in Glasgow, UK, during the plenary session (10:30 - 12:30) on Monday, 1 June 2020. www.hypertension2020.org

Make sure to attend this highlight of the congress!

Alta E. Schutte (President ISH) Thomas Unger (Chair, ISH Guidelines Committee) 